\title{
Nuevo método para elaborar corredores endémicos
}

\author{
Pablo Wenceslao Orellano ${ }^{1}$ y Julieta Itatí Reynoso ${ }^{2}$
}

Forma de citar Orellano PW, Reynoso JI. Nuevo método para elaborar corredores endémicos. Rev Panam Salud Publica. 2011;29(5):309-14.

RESUMEN Objetivo. Diseñar un instrumento para elaborar corredores o canales endémicos mediante cálculos sencillos y, en el caso de enfermedades transmisibles, identificar y estimar parámetros relacionados con la dinámica de la transmisión.

Métodos. Se obtuvieron parámetros para elaborar una curva teórica de incidencia esperada, basada en el modelo logístico de crecimiento. Los parámetros se estimaron por medio de una regresión no lineal, a partir de datos de incidencia acumulada de los cinco años anteriores. Se tomó como ejemplo la incidencia semanal acumulada de casos de enfermedad tipo influenza para Argentina en 2009 y se la comparó con la serie de casos de 2004 a 2008 para construir los canales endémicos acumulados y no acumulados.

Resultados. De acuerdo a los canales endémicos acumulados obtenidos, el país entró en zona de brote a partir de la semana dos. El máximo número esperado de casos o capacidad de carga $(K)$ de acuerdo a los datos de años previos fue de 1090 660. Considerando los corredores no acumulados, la situación de brote se presentó en 34 de las 51 semanas. Se estimó un rango para la tasa básica de reproducción $\left(R_{0}\right)$ de 1,05 a 1,13 para el período no epidémico 2004-2008.

Conclusiones. El nuevo método permitió elaborar canales endémicos de una manera sencilla, con la obtención de parámetros importantes para la transmisión. Si bien tiene limitaciones, entre ellas que la ecuación utilizada es más útil para evaluar enfermedades con un ciclo anual marcado y menos exacta para ciclos menores de un año, puede ser considerado como una alternativa valiosa para elaborar corredores endémicos y una nueva contribución al estudio de brotes epidémicos en los niveles locales de vigilancia de la salud.

Palabras clave Modelos epidemiológicos; técnicas de apoyo para la decisión; brotes de enfermedades; subtipo H1N1 del virus de la influenza A; Argentina.

Los corredores o canales endémicos son una herramienta de amplio uso en vigilancia epidemiológica que sirve para establecer los valores esperados de incidencia de una enfermedad en un tiempo y un lugar determinados. Estos valores se fijan a partir del registro de una serie temporal

\footnotetext{
Departamento de Epidemiología, Ministerio de Salud de Tierra del Fuego, Antártida e Islas del Atlántico Sur, Argentina. La correspondencia se debe dirigir a Pablo Wenceslao Orellano. Correo electrónico: porellano@gmail.com

2 Dirección de Epidemiología, Ministerio de Salud de la Nación, Buenos Aires, Argentina.
}

de 5 a 10 años previos al año que se desea evaluar, con valores medios y rangos o intervalos calculados para una unidad de tiempo determinada, que usualmente es de 7 días y se define como semana epidemiológica. Cuando la curva de incidencia del año en curso sobrepasa el valor esperado o habitual de casos, se considera que hay brote o epidemia.

De los distintos métodos que se han desarrollado para elaborar corredores endémicos, el más simple consiste en diagramar el número máximo y mínimo de casos, y confeccionar de esta manera una banda, por encima de la cual se considera en área de brote. Pueden utilizarse también la mediana y los cuartiles de la incidencia por unidad de tiempo (meses o semanas), para generar una figura con cuatro áreas definidas como zona de éxito, de seguridad, de alerta y de epidemia. Se puede encontrar una adecuada introducción a la evolución de estos métodos en el estudio de Bortman, donde además se desarrolla un método numérico más complejo para generar con datos empíricos un gráfico con las cuatro áreas mencionadas (1). Este método incorpora 
las medias geométricas de las tasas y su desviación estándar para estimar los intervalos de confianza que determinarán los valores extremos. ${ }^{3}$ Desde su publicación original en 1999 y hasta la actualidad, el modelo de Bortman ha significado un invaluable aporte a la vigilancia epidemiológica para el monitoreo de situaciones de brotes epidémicos, y es utilizado regularmente por las oficinas locales y nacionales que analizan datos de salud en Argentina y otros países de la Región.

La confección de una curva esperada de casos a partir de datos empíricos de incidencia de años previos, sin realizar supuestos teóricos que permitan identificar parámetros, implica que la curva esperada de incidencia (con sus intervalos de confianza) puede tener incluidos valores relacionados con fluctuaciones estacionales, mensuales o de períodos de tiempo variables, que no necesariamente representan variaciones relevantes para la dinámica de la enfermedad. Es decir que los métodos enunciados generan curvas que se encuentran sujetas a la posibilidad de grandes variaciones en la incidencia entre una semana y la siguiente, dificultando así el análisis y la interpretación.

En un estudio sobre mortalidad por neumonía e influenza publicado en 1967, Serfling y Sherman aplicaron un método para la confección de canales endémicos que considera el exceso de mortalidad como un indicador de epidemia, construyendo la curva de valores esperados de mortalidad con sustento teórico y de un modo más complejo (2). Este modelo discrimina la tendencia secular mediante un término lineal y las variaciones estacionales con términos que incluyen senos y cosenos, excluyendo la variación aleatoria de los canales endémicos. Tal construcción teórica permite elaborar canales más precisos, toda vez que reduce la confusión debida a variaciones aleatorias sin importancia para la transmisión. Martínez y colaboradores, por su parte, presentan un enfoque de vigilancia epidemiológica que diseña canales endémicos a partir de una modificación del modelo estadístico de Serfling (3). Este método, a diferencia del de Bortman, tiene la limitación de requerir una capacidad de cálculo avanzado, con progra-

\footnotetext{
Este método se puede utilizar a través de hojas de cálculo de Excel y mediante el Epidat, programa de distribución libre desarrollado por la Consellería de Sanidade de la Xunta de Galicia y la Organización Panamericana de la Salud (OPS) para el análisis epidemiológico.
}

mas no siempre disponibles por las unidades locales de vigilancia encargadas de analizar los datos.

En realidad ninguno de los modelos empíricos mencionados permite estimar parámetros que expliquen la dinámica de transmisión de una enfermedad. Por ejemplo, no revelan la tasa básica de reproducción $\left(\mathrm{R}_{0}\right)$, parámetro que representa el número medio de casos secundarios de la enfermedad que pueden iniciarse por contagio a partir de una sola persona infectada. Si el $\mathrm{R}_{0}$ es menor que 1 , cada persona infectada generará en promedio menos de una nueva persona infectada, por lo cual puede estimarse que la infección tenderá a la extinción. Si el $\mathrm{R}_{0}$ es mayor que 1 , la enfermedad tiene la capacidad de expandirse entre la población susceptible. En este sentido, el objetivo del presente trabajo fue diseñar un instrumento para elaborar corredores o canales endémicos mediante cálculos sencillos y, en el caso de las enfermedades transmisibles, identificar y estimar parámetros relacionados con la dinámica de la transmisión.

\section{MATERIALES Y MÉTODOS}

\section{Elaboración de la curva teórica de casos acumulados}

El método utiliza como datos de entrada la incidencia acumulada a la semana $t$ de la enfermedad en estudio durante el año que se desea evaluar $\left(\mathrm{A}_{\mathrm{x}(\mathrm{t})}\right) \mathrm{y}$ los 5 años anteriores $\left(A_{x t-1(t)} \ldots A_{x-5(t)}\right)$. En un primer paso se construye la incidencia acumulada esperada $\left(\mathrm{AE}_{\mathrm{x}(\mathrm{t})}\right)$ del año a evaluar, para cada semana, como el promedio de la incidencia en los 5 años previos considerados, según la siguiente ecuación:

$$
\begin{aligned}
A E_{x(t)}= & \left(A_{x-5(t)}+A_{x-4(t)}+A_{x-3(t)}+A_{x-2(t)}+\right. \\
& \left.A_{x-1(t)}\right) / 5
\end{aligned}
$$

Esta incidencia $\mathrm{AE}_{\mathrm{x}(\mathrm{t})}$ se calcula para cada semana epidemiológica $\left(\mathrm{AE}_{\mathrm{x}, \mathrm{t}=1^{\prime}}\right.$, $\left.\mathrm{AE}_{\mathrm{x}, \mathrm{t}=2}, \ldots . \mathrm{AE}_{\mathrm{x}, \mathrm{t}=52}\right)$. El conjunto de valores $\mathrm{AE}_{\mathrm{x}(\mathrm{t})}$ constituye la curva esperada de incidencia acumulada, basada en datos empíricos. Luego, con estos valores se elabora la curva teórica de incidencia, de acuerdo a un modelo de crecimiento logístico que considere la tasa intrínseca de crecimiento del número de casos $\left(\mathrm{r}_{0}\right)$. Para ello, en el presente estudio se utilizó la ecuación de crecimiento logístico de Verhulst-Pearl, citada por
Tsoularis, modelo que ha conformado históricamente la base de otros modelos más generalizados (4):

$\mathrm{TA}_{(\mathrm{t})}=\mathrm{K} \times \mathrm{TA}_{0} /\left(\left(\mathrm{K}-\mathrm{TA}_{0}\right) \mathrm{e}^{-\mathrm{r} 0 \times \mathrm{t}}+\mathrm{TA}_{0}\right)$

Esta curva estima una tasa teórica acumulada en la semana $t\left(\mathrm{TA}_{(\mathrm{t})}\right)$, desde la semana 1 a la 52, a partir de los parámetros $\mathrm{r}_{0}, \mathrm{TA}_{0} \mathrm{y} \mathrm{K}$, los cuales representan la incidencia inicial de casos (semana cero) y la incidencia máxima posible. Se utiliza la letra " $\mathrm{K}$ " para el parámetro de incidencia máxima posible debido a su equivalencia con el concepto de capacidad de carga, usado en estudios de ecología de poblaciones (5). Los parámetros $\mathrm{r}_{0}, \mathrm{TA}_{0} \mathrm{y}$ $\mathrm{K}$ pueden ser estimados a partir de la curva de incidencia esperada por semana $\left(\mathrm{AE}_{\mathrm{x}(\mathrm{t})}\right)$ mediante un modelo de regresión no lineal, utilizando un programa estadístico como el SPSS o el R software; ambos utilizan el algoritmo de Levenberg-Marquardt (6).

Una alternativa de más fácil acceso es utilizar el componente Solver del programa Excel de Microsoft, que usa un código de optimización no lineal (método GRG2) (7). Con objeto de facilitar la aplicación del método, los autores han desarrollado un archivo del programa Excel, versión 97 o posterior, que permite realizar los cálculos en forma sencilla e incluye una macro para estimar los corredores de manera automática, así como un instructivo para optar por el sistema no automático. ${ }^{4}$

Dado que en todos los programas la resolución de la ecuación no lineal se alcanza por medio de un procedimiento iterativo, los valores iniciales de los parámetros deben proveerse siempre al inicio de la primera iteración. Si bien en la mayoría de los escenarios cualquier valor inicial de los parámetros puede funcionar correctamente, se propone utilizar valores iniciales estándar con el fin de uniformar las estimaciones finales. Estos valores corresponden a la incidencia esperada en la semana 1 y en la semana 52, y una tasa intrínseca de crecimiento nula, lo que equivaldría a un $R_{0}=1$ (sin expansión de la enfermedad):

$$
\begin{aligned}
& \mathrm{K}_{\text {inicial }}=\mathrm{AE}_{\mathrm{x}, \mathrm{t}=52} \\
& \mathrm{TA}_{0 \text { inicial }}=\mathrm{AE}_{\mathrm{x}, \mathrm{t}=1} \\
& \mathrm{r}_{0 \text { inicial }}=0
\end{aligned}
$$

4 Estos archivos pueden solicitarse por correo electrónico al autor de correspondencia. 


\section{Cálculo de los intervalos de confianza para la curva}

La curva obtenida representa una estimación de la incidencia teórica de casos por semana, a partir de la incidencia observada en los 5 años anteriores, y está determinada por los valores de tres parámetros: $\mathrm{r}_{0}, \mathrm{TA}_{0} \mathrm{y} \mathrm{K}$. El primero, la tasa intrínseca de crecimiento, corresponde a la velocidad de aparición de nuevos casos de la enfermedad y en el presente método se supone como invariante para la obtención de curvas que representen los intervalos de confianza inferior y superior respecto al valor medio. Por lo tanto, para la confección de estas curvas se variarán los valores de los parámetros $\mathrm{TA}_{0} \mathrm{y} \mathrm{K}$, que corresponden al número inicial de casos y al número máximo posible. De esta manera, pueden reconocerse tres curvas teóricas de incidencia:

$$
\begin{aligned}
& \mathrm{TA}_{(\mathrm{t})}=\mathrm{K} \times \mathrm{TA}_{0} /\left(\left(\mathrm{K}-\mathrm{TA}_{0}\right) \mathrm{e}^{-\mathrm{r} 0 \times \mathrm{t}}+\mathrm{TA}_{0}\right) \\
& \mathrm{TA}^{\sup }{ }_{(\mathrm{t})}=\mathrm{K}^{\text {sup }} \times \mathrm{TA}_{0} \text { sup } /\left(\left(\mathrm{K}^{\text {sup }}-\mathrm{TA}_{0}{ }^{\text {sup }}\right)\right. \\
& \mathrm{e}^{-\mathrm{r} 0 \times \mathrm{t}}+\mathrm{TA}_{0}^{\text {sup })} \\
& \mathrm{TA}^{\text {inf }}=\mathrm{K}^{\text {inf }} \times \mathrm{TA}_{0}{ }^{\text {inf }} /\left(\left(\mathrm{K}^{\text {inf }}-\mathrm{TA}_{0}^{\text {inf }}\right) \mathrm{e}^{-\mathrm{r} 0}\right. \\
& \left.\times \mathrm{t}+\mathrm{TA}_{0}^{\text {inf }}\right)
\end{aligned}
$$

Las últimas dos ecuaciones dependen de la obtención de los nuevos parámetros $\mathrm{TA}_{0}^{\text {sup }}, \mathrm{K}^{\text {sup }}, \mathrm{TA}_{0}{ }^{\text {inf }} \mathrm{y} \mathrm{K}^{\text {inf }}$, ya que el parámetro $\mathrm{r}_{0}$ se mantiene constante para las tres curvas.

Para obtener los parámetros $\mathrm{TA}_{0}$ y K superior e inferior $\left(\mathrm{TA}_{0}\right.$ sup, $\mathrm{TA}_{0}$ inf, $\mathrm{K}^{\text {sup }} \mathrm{y} \mathrm{K}^{\mathrm{inf}}$ ) a partir de los valores de $\mathrm{TA}_{0}$ y K antes estimados, se utilizó el mismo concepto que en la construcción de umbrales epidémicos del modelo estadístico de Serfling (2), que crea un límite superior equivalente al valor estimado más 1,64 desviaciones estándar (DE) de los años anteriores considerados, un valor que la experiencia ha demostrado útil para distinguir los incrementos epidémicos de la variación aleatoria. Los parámetros $\mathrm{TA}_{0} \mathrm{y} \mathrm{K}$ superior e inferior se obtienen de la siguiente forma:

$$
\begin{aligned}
& \mathrm{K}^{\text {sup }}=\mathrm{K}+\left(1,64 \times \mathrm{DE}_{\mathrm{t}=52}\right) \\
& \mathrm{K}^{\text {inf }}=\mathrm{K}-\left(1,64 \times \mathrm{DE}_{\mathrm{t}=52}\right) \\
& \mathrm{TA}_{0} \text { sup }=\mathrm{TA}_{0}+\left(1,64 \times \mathrm{DE}_{\mathrm{t}=1}\right) \\
& \mathrm{TA}_{0}{ }^{\text {inf }}=\mathrm{TA}_{0}-\left(1,64 \times \mathrm{DE}_{\mathrm{t}=1}\right)
\end{aligned}
$$

Para estimar los parámetros $\mathrm{TA}_{0}$ superior e inferior se utiliza la menor DE de la curva de incidencia esperada, que co- rresponde a la semana $1\left(\mathrm{DE}_{\mathrm{t}=1}\right)$, mientras que para los parámetros $\mathrm{K}$ superior e inferior se utiliza la semana $52\left(\mathrm{DE}_{\mathrm{t}=52}\right)$, que es la de mayor DE.

\section{Construcción de los canales endémicos acumulados}

Para la construcción de los canales, se utilizan los valores teóricos de $\mathrm{TA}_{(\mathrm{t})}$, $\mathrm{TA}_{(\mathrm{t})}$ superior y $\mathrm{TA}_{(\mathrm{t})}$ inferior. Estas tres curvas dan lugar a la conformación de cuatro zonas, utilizándose las mismas que en el estudio de Bortman (1):

Zona de éxito: por debajo de la curva inferior.

Zona de seguridad: entre la curva inferior y la media.

Zona de alerta: entre la curva media y la superior.

Zona de epidemia: por encima de la curva superior.

Para determinar si, en una semana dada, un área se encuentra ante una situación de epidemia, la curva de casos acumulados observada para el año en estudio debe estar por encima de la curva teórica superior.

\section{Cálculo de parámetros clave para la transmisión}

La elaboración de curvas teóricas permite, además de obtener canales endémicos, estimar parámetros que revisten gran importancia para la caracterización de la dinámica de las enfermedades transmisibles en un sitio y un tiempo determinados. Dos de los parámetros estimados — $\mathrm{el} \mathrm{TA}_{0}$ y el $\mathrm{K}$ - representan, respectivamente, el número de casos con que comienza la notificación de la enfermedad en el año y el número máximo de casos acumulados que es posible alcanzar. Asimismo, el parámetro $\mathrm{r}_{0}$ (tasa intrínseca de crecimiento) representa la velocidad con que aumenta el número de casos a lo largo del año en base semanal (semanas ${ }^{-1}$ ). A partir de este parámetro, y conociendo el intervalo serial (IS) de la enfermedad, se puede calcular el número reproductivo básico o tasa reproductiva básica $\left(R_{0}\right)$. La relación entre $\mathrm{r}_{0}$, IS y $\mathrm{R}_{0}$ puede observarse en la siguiente ecuación (8):

$\mathrm{R}_{0}=\mathrm{e}^{(\mathrm{r} 0 \times \mathrm{IS})}$

Es necesario señalar que la tasa intrínseca de crecimiento y el IS deben tener unidades comparables entre sí (p. ej. semanas o días).

\section{Alternativas del modelo para la elaboración de canales endémicos}

El mismo método puede ser utilizado para distintas escalas temporales (p. ej. en base mensual o cuatri-semanal) así como también pueden utilizarse - para la estimación de la incidencia- los promedios de 7 ó 10 años previos. Más aún, es posible realizar los canales endémicos para la incidencia semanal no acumulada en lugar de la acumulada, simplemente restando los valores de $\mathrm{AE}_{\mathrm{x}} \mathrm{y} \mathrm{TA}_{(\mathrm{t})}$ entre una semana y la semana previa. Este cálculo genera los canales endémicos a partir de la semana 2 y hasta la 52 .

\section{Aplicación del método en una enfermedad tipo influenza}

Para ejemplificar el uso del modelo, se evaluó la transmisión de enfermedad tipo influenza de acuerdo a las notificaciones del sistema nacional de vigilancia epidemiológica de Argentina durante 2009, cuando la pandemia de influenza H1N1 afectó a todas las provincias de este país y el nuevo virus dio cuenta de $80 \%$ de todos los casos de influenza notificados (9). Los canales endémicos fueron elaborados utilizando los casos acumulados notificados entre 2004 y 2008 en el mismo sistema. Los cálculos se realizaron en el programa Excel, con el archivo desarrollado por los autores para tal fin. Además de los canales endémicos acumulativos, se realizaron los canales de incidencia semanal no acumulada para la misma enfermedad y los mismos períodos de tiempo considerados. Se realizó un análisis de consistencia entre el nuevo método y el modelo de Bortman, considerando el número de semanas detectadas por cada procedimiento como elevadas por encima del umbral de brote epidémico (semanas en brote), en los corredores de incidencia semanal no acumulada. Se calculó el índice kappa mediante el programa Epidat 3.0.

\section{RESULTADOS}

De acuerdo a los datos notificados por el sistema nacional de vigilancia epidemiológica de Argentina para 2004-2008, se estimó una curva teórica esperada con un número inicial $\left(\mathrm{TA}_{0}\right.$ ) de 7708 casos de enfermedad tipo influenza y un má- 
FIGURA 1. Casos acumulados de enfermedad tipo influenza para 2009 y corredores endémicos para 2004-2008, Argentina

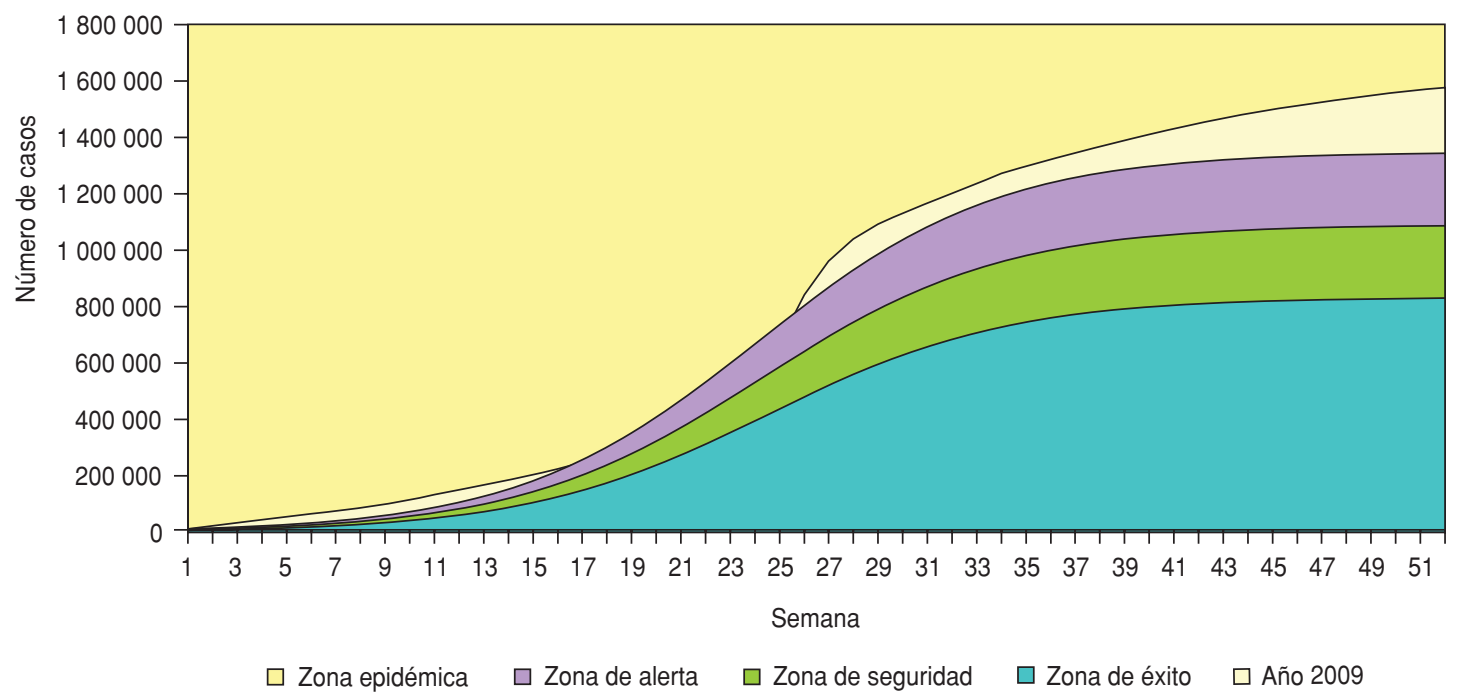

Fuente: Sistema Nacional de Vigilancia Epidemiológica, Argentina.

ximo posible de casos acumulados $(\mathrm{K})$ de 1090 660. En las figuras 1 y 2 se presentan los resultados gráficos de los corredores endémicos generados por el nuevo método, tanto para la incidencia acumulada como para la no acumulada. La tasa intrínseca de crecimiento $\left(\mathrm{r}_{0}\right)$ se estimó en 0,20; como no se contó con el valor del intervalo serial, se utilizó un rango de 1,6-4,3 días (ó 0,23-0,61 semanas) de acuerdo con datos de fuentes bibliográficas y de estimaciones teóricas y empíricas realizadas sobre ese parámetro (10-17).
Con estos valores se calculó una tasa reproductiva básica $\left(R_{0}\right)$ en un rango de 1,05 a 1,13. En el cuadro 1 se observan los valores obtenidos para los diferentes parámetros con el nuevo método, comparados con los calculados por el modelo de Bortman. Al utilizar el criterio planteado - por el nuevo método- para evaluar las diferentes zonas de riesgo indicadas por la curva de incidencia acumulada, el país se encontró en situación de brote epidémico en la semana número dos. Cuando se consideraron los corredores endémicos no acumulados, la situación de brote se observó en 34 de las 51 semanas analizadas. Tomando en cuenta el número de semanas consideradas bajo situación de brote para uno y otro método en la curva de incidencia no acumulada, se calculó un índice kappa de -0,05 (intervalo de confianza de 95\%: $-0,26-0,17$ ) (cuadro 2).

\section{DISCUSIÓN}

El nuevo método permitió elaborar los corredores endémicos de enfermedad

FIGURA 2. Casos no acumulados de enfermedad tipo influenza para 2009 y corredores endémicos para 2004-2008, Argentina

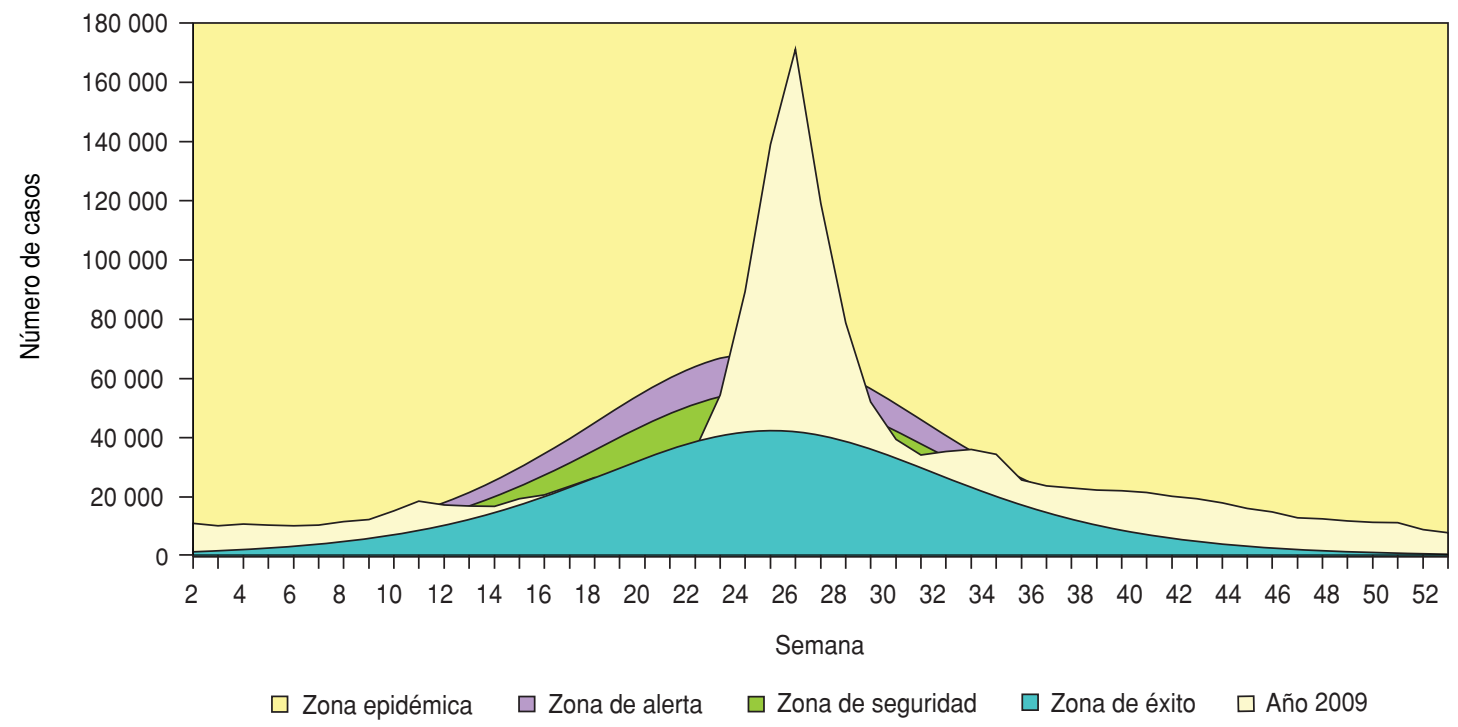

Fuente: Sistema Nacional de Vigilancia Epidemiológica, Argentina. 
CUADRO 1. Parámetros e intervalos de los corredores endémicos de enfermedad tipo influenza, según el método de Bortman y el del presente estudio, Argentina, 2004-2008

\begin{tabular}{lrr}
\hline \multicolumn{1}{c}{ Parámetro/Intervalo } & \multicolumn{2}{c}{ Método } \\
\cline { 2 - 3 } & \multicolumn{1}{c}{ Bortman } & Presente estudio \\
\hline Número de casos en la semana uno & 8967 & 937 \\
Número de casos en la semana 52 & 1113128 & 1086866 \\
Número de casos en la semana cero (TA $\left.{ }_{0}\right)$ & $\mathrm{NA}^{\mathrm{a}}$ & 7708 \\
Número máximo posible de casos $(\mathrm{K})$ & $\mathrm{NA}^{\mathrm{a}}$ & 1090660 \\
Intervalo de incidencia del umbral epidémico & & $12035-1343777$ \\
$\quad$ (incidencia acumulada) & $10430-1300539$ & 2 \\
Semana de entrada en la zona de epidemia & & 0,20 \\
$\quad$ (incidencia acumulada) & 1 & $(1,05-1,13)$ \\
Tasa intrínseca de crecimiento $\left(\mathrm{r}_{0}\right)$ (semanas $\left.{ }^{-1}\right)$ & $\mathrm{NA}^{\mathrm{a}}$ & $\mathrm{NA}^{\mathrm{a}}$ \\
Tasa reproductiva básica $\left(\mathrm{R}_{0}\right)$ & & \\
\hline
\end{tabular}

Fuente: elaborado por los autores.

a NA: los datos no son aplicables.

tipo influenza de Argentina de una manera sencilla, facilitando la obtención de parámetros de relevancia para la transmisión de la enfermedad. Para el análisis de la incidencia acumulada de 2009, el nuevo método indicó la entrada en la zona epidémica con una semana de retraso respecto del método de Bortman, lo que implica una menor sensibilidad en los momentos iniciales de la transmisión. Por el contrario, al tener en cuenta los corredores para la incidencia no acumulada, se detectaron 18 semanas más que las estimadas por el método de Bortman. De acuerdo al valor del índice kappa calculado, la consistencia entre ambos procedimientos es pobre, reforzando la idea de que la información aportada difiere entre ambos (18).

La obtención de las curvas teóricas permite indicar límites sin variaciones aleatorias, cuyo efecto es más evidente en la figura de incidencia semanal no acumulada. Mientras que el nuevo método permite confeccionar curvas en forma de campana, el modelo de Bortman lleva a la obtención de curvas con bordes aserrados, lo que podría ser consecuencia de patrones aleatorios.

A través del modelo de Bortman es posible estimar el número esperado de casos acumulados en la semana 1 y en la
52 - la última del año. Con el nuevo método, además de esta estimación, también se pueden obtener dos parámetros de naturaleza más teórica o conceptual, como son el número de casos a la semana cero $\left(\mathrm{TA}_{0}\right)$ y el máximo número posible de casos (K) o capacidad de carga máxima de casos para el lugar, de acuerdo a la curva teórica. Adicionalmente, el nuevo método permitió calcular la tasa intrínseca de crecimiento $\left(\mathrm{r}_{0}\right)$ y el número reproductivo básico $\left(\mathrm{R}_{0}\right)$.

Sobre el segundo parámetro, la literatura ofrece diversas estimaciones realizadas tanto de modo teórico como empírico para la influenza. Según estas fuentes, el $\mathrm{R}_{0}$ estaría comprendido en un rango de 1,2 y $2,0(13,14,19-23)$. Sin embargo, estas estimaciones fueron hechas con datos de incidencia en el contexto de brotes epidémicos, muchos de ellos durante la pandemia de influenza $\mathrm{H} 1 \mathrm{~N} 1 \mathrm{de}$ 2009 , por lo cual estos valores de $R_{0}$ no deberían ser considerados como parámetros representativos para un corredor endémico. De hecho, el menor de estos valores $\left(R_{0}=1,2\right)$ fue estimado durante un brote en la comunidad de La Gloria, México, y correspondería al menor valor de $\mathrm{R}_{0}$ observado hasta el momento que permite la existencia de un brote (13). Estos datos son consistentes con la estimación

CUADRO 2. Comparación del número de semanas consideradas en situación de brote de enfermedad tipo influenza para 2009 según el método de Bortman y el del presente estudio, utilizando los corredores 2004-2008 para incidencia no acumulada, Argentina

\begin{tabular}{l|lcc}
\hline \multirow{2}{*}{} & & \multicolumn{2}{c}{ Método de Bortman } \\
\cline { 3 - 4 } & & En brote & Fuera de brote \\
\hline \multirow{2}{*}{ Presente estudio } & En brote & 10 & 24 \\
\cline { 2 - 4 } & Fuera de brote & 6 & 11 \\
\hline
\end{tabular}

Fuente: elaborado por los autores. del presente modelo de un $\mathrm{R}_{0}$ comprendido entre 1,05 y 1,13 para años no epidémicos, y reforzaría la idea de que con un $\mathrm{R}_{0}$ superior se daría paso a una situación de brote epidémico.

La metodología aquí propuesta tiene sin embargo varias limitaciones. En primer lugar, la posibilidad de que la ecuación de la curva logística empleada no sea la que mejor describa la dinámica de incidencia acumulada de la enfermedad en estudio. En este sentido, la ecuación es más útil para evaluar enfermedades con un ciclo anual marcado, mientras que sería menos exacta para ciclos menores a un año. Por otro lado, la estimación del parámetro $\mathrm{R}_{0}$ a partir del $\mathrm{r}_{0}$ tiene implícitas limitaciones, como las fluctuaciones estocásticas de la incidencia durante los comienzos de la transmisión y el hecho de que esta estimación dependa fuertemente del modelo de cálculo utilizado para relacionar ambos parámetros (8).

La resolución de la ecuación no lineal planteada por el método presenta además variaciones relacionadas con la forma en que se obtienen los parámetros, dado que los métodos iterativos dependen muchas veces de los parámetros iniciales elegidos para correr el modelo y de los algoritmos de cálculo utilizados. Dado que variaciones en estos puntos pueden llevar a diferencias en los parámetros obtenidos, es importante la estandarización de los procedimientos empleados. La utilización de ecuaciones no lineales presenta también otras dificultades, ya que obliga a un mayor desarrollo matemático y al empleo de programas estadísticos más específicos, que no son accesibles en forma gratuita ni son de uso común en los niveles locales encargados de los análisis de vigilancia. A este respecto, la elaboración de un archivo para utilizar en el programa Microsoft Excel representa una ayuda importante, siendo este de amplia distribución. Es esperable que si el método es juzgado como una herramienta útil para el apoyo de los sistemas de vigilancia, sea posible su incorporación en programas de distribución libre y uso común en vigilancia epidemiológica como el Epidat $\mathrm{u}$ otros. No obstante, aun con las limitaciones enumeradas, este método puede ser considerado como una alternativa valiosa para la elaboración de corredores endémicos respecto a otros modelos y una nueva contribución al estudio de brotes epidémicos en los niveles locales de vigilancia de la salud. 


\section{REFERENCIAS}

1. Bortman M. Elaboración de corredores o canales endémicos mediante planillas de cálculo. Rev Panam Salud Publica. 1999;5(1):1-8.

2. Serfling RE, Sherman IL, Houseworth WJ. Excess pneumonia-influenza mortality by age and sex in three major influenza A2 epidemics, United States, 1957-58, 1960 and 1963. Am J Epidemiol. 1967;86(2):433-41.

3. Martínez R, Alonso A, Díaz JC, Comas T, Castañeda JO. Vigila: un instrumento para la vigilancia epidemiológica de enfermedades transmisibles. Rev Cubana Med Trop. 1999; 51(1):38-45.

4. Tsoularis A, Wallace J. Analysis of logistic growth models. Math Biosci. 2002;179(1): 21-55.

5. Sayre NF. The genesis, history, and limits of carrying capacity. Ann Assoc Am Geogr. 2008;98(1):120-34.

6. Seber GA, Wild CJ. Nonlinear Regression. New Jersey: John Wiley and Sons; 2003.

7. Cornell P. Beginning Excel What-If Data Analysis Tools: Getting Started with Goal Seek, Data Tables, Scenarios, and Solver. New York: Springer-Verlag; 2006.

8. Wallinga J, Lipsitch M. How generation intervals shape the relationship between growth rates and reproductive numbers. Proc Biol Sci. 2007;274(1609):599-604.

9. Zala C, Gonzalez R. Respiratory disease in adults during pandemic (H1N1) 2009 outbreak, Argentina. Emerg Infect Dis. 2009; 15(12):2060-1.
10. Lessler J, Cummings DA, Fishman S, Vora A, Burke DS. Transmissibility of swine flu at Fort Dix, 1976. J R Soc Interface. 2007;4(15):755-62.

11. White LF, Pagano M. Transmissibility of the influenza virus in the 1918 pandemic. PLoS One. 2008;3(1):e1498.

12. Cowling BJ, Fang VJ, Riley S, Malik Peiris JS, Leung GM. Estimation of the serial interval of influenza. Epidemiology. 2009;20(3):344-7.

13. Fraser C, Donnelly CA, Cauchemez S, Hanage WP, Van Kerkhove MD, Hollingsworth TD, et al. Pandemic potential of a strain of influenza A (H1N1): early findings. Science. 2009;324(5934):1557-61.

14. Yang Y, Sugimoto JD, Halloran ME, Basta NE, Chao DL, Matrajt L, et al. The transmissibility and control of pandemic influenza A (H1N1) virus. Science. 2009;326(5953):729-33.

15. Ghani AC, Baguelin M, Griffin J, Flasche S, Pebody R, van Hoek AJ, et al. The Early Transmission Dynamics of H1N1pdm Influenza in the United Kingdom. PLoS Curr. 2009;1:RRN1130.

16. White LF, Wallinga J, Finelli L, Reed C, Riley $\mathrm{S}$, Lipsitch M, et al. Estimation of the reproductive number and the serial interval in early phase of the 2009 influenza A/H1N1 pandemic in the USA. Influenza Other Respi Viruses. 2009;3(6):267-76.

17. Lessler J, Reich NG, Cummings DA; New York City Department of Health and Mental Hygiene Swine Influenza Investigation Team, Nair HP, Jordan HT, et al. Outbreak of 2009 pandemic influenza A (H1N1) at a New York City school. N Engl J Med. 2009;361(27): 2628-36.

18. Fleiss JL. Statistical Methods for Rates and Proportions. 2. ${ }^{\text {a }}$ ed. New York: John Wiley; 1981.

19. Vynnycky E, Edmunds WJ. Analyses of the 1957 (Asian) influenza pandemic in the United Kingdom and the impact of school closures. Epidemiol Infect. 2008;136(2):166-79.

20. Caley P, Philp DJ, McCracken K. Quantifying social distancing arising from pandemic influenza. J R Soc Interface. 2008;5(23):631-9.

21. Flahault A, Vergu E, Boëlle PY. Potential for a global dynamic of Influenza A (H1N1). BMC Infect Dis. 2009;9:129.

22. Balcan $\mathrm{D}, \mathrm{Hu} \mathrm{H}$, Goncalves $\mathrm{B}$, Bajardi $\mathrm{P}$, Poletto C, Ramasco JJ, et al. Seasonal transmission potential and activity peaks of the new influenza $\mathrm{A}(\mathrm{H} 1 \mathrm{~N} 1)$ : a Monte Carlo likelihood analysis based on human mobility. BMC Med. 2009;7:45.

23. Tuite AR, Greer AL, Whelan M, Winter AL, Lee B, Yan P, et al. Estimated epidemiologic parameters and morbidity associated with pandemic H1N1 influenza. CMAJ. 2010;182(2): $131-6$.

Manucrito recibido el 12 de agosto de 2010. Aceptado para publicación, tras revisión, el 3 de febrero de 2011.

ABSTRACT Objective. Design an instrument for determining endemic levels or ranges using simple calculations; identify and estimate the parameters related to the dynamic transmission of communicable diseases.

New method for determining endemic levels

Key words
Methods. The parameters for establishing a theoretical curve of expected incidence based on the logistic growth model were identified. The parameters were estimated by nonlinear regression based on the cumulative incidence data from the previous five years. The weekly cumulative incidence of cases of influenza-like illness in Argentina in 2009 was used as an example. It was compared to the 2004-2008 case series in order to determine the cumulative and non-cumulative endemic levels.

Results. According to the cumulative endemic levels identified, the country entered the outbreak area in week 2 . The data from previous years showed that the maximum expected number of cases or carrying capacity (K) was 1090 660. When the non-cumulative levels were considered, the outbreak was present in 34 out of 51 weeks. A range of 1.05 to 1.13 was estimated for the basic reproductive rate $\left(R_{0}\right)$ in the non-epidemic period from 2004-2008.

Conclusions. The new method facilitated the determination of endemic levels using a simple procedure with the identification of parameters that are important for transmission. Although it has limitations such as the fact that the equation used is more appropriate for evaluating diseases with a pronounced annual cycle and less accurate for cycles of less than 1 year, it can be considered a valuable alternative method for determining endemic ranges and a new contribution to the study of epidemic outbreaks at local health surveillance levels.

Epidemiologic models; decision support techniques; disease outbreaks; influenza A virus, H1N1 subtype; Argentina. 